\title{
Wayne's assay: a screening method for indirect detection of pyrazinamide resistance in Mycobacterium tuberculosis clinical isolates
}

\begin{abstract}
Pyrazinamide (PZA) is often used for treatment of tuberculosis (TB) and PZA in combination with isoniazid (INH) is rapidly bactericidal for replicating and nonreplicating forms of Mycobacterium tuberculosis (MTB) strains. MTB produces a pyrazinamidase (PZase) enzyme and most PZA resistant strains lack this enzyme. The lack of PZase activity and it's correlation with PZA resistance has been associated with mutations in the pncA gene which encodes the PZase. The enzymatic pyrazinamidase assay by Wayne's method for determining PZase activity can be used as a screening method for PZA drug susceptibility testing (DST), since resistant strains are PZase negative. The gold standard for detection of PZA resistance is PCR mediated direct DNA sequencing using Sanger method. In this study, 343 strains of MTB were tested by both Wayne's method and Sanger method. The sensitivity and specificity of Wayne's was $28.37 \%$ and $37.78 \%$, respectively, with PPV of $41.26 \%$ and NPV of $25.50 \%$. Our findings suggest that Wayne's method can be used as a screening test for the detection of PZA resistance..
\end{abstract}

Keywords: wayne's method, mycobacterium tuberculosis, pyrazinamide, pnca, sanger sequencing
Volume 5 Issue I - 2017

\author{
Vaishali R Wabale,' Ameeta A Joshi,' \\ Muthuraj Muthaiah, ${ }^{2}$ Abhay S Chowdhary' \\ 'Department of Microbiology, Grant Government Medical \\ College, India \\ ${ }^{2}$ Laboratory Director and In charge, Intermediate Reference \\ Laboratory, India
}

Correspondence: Vaishali R Wabale, Assistant Professor Department of Microbiology, Grant Government Medical College \& Sir JJ Hospitals, Byculla, Mumbai, Maharashtra, Pin code - 400008, India, Tel 8828050378

Email vrwabale@gmail.com

Received: June 01, 2017 | Published: July 12, 2017
Abbreviations: TB, tuberculosis; MTB, mycobacterium tuberculosis; MDR, multidrug-resistant; DST, drug susceptibility testing; POA, pyrazinoic acid; NTM, non-tuberculous mycobacterium

\section{Introduction}

Tuberculosis (TB) caused by members of Mycobacterium tuberculosis (MTB) complex is one of the common lung diseases, causing 3,000,000 deaths per year worldwide. ${ }^{1}$ This global epidemic has become more dangerous due to the presence of HIV/AIDS especially in developing countries, the emergence of multidrugresistant (MDR)TB and recently described extensively drug-resistant TB. ${ }^{1}$ Timely detection of drug resistance in patients with TB is a priority and reliable methods for rapid drug susceptibility testing (DST) are needed for better control of the disease. ${ }^{2}$ The increasing number of drug-resistant MTB strains has made the rapid identification of susceptibility clinically important because patients suffering from infections due to these strains require specialized antibiotic treatment. Anti-mycobacterial drug susceptibility testing (DST) is necessary for the proper treatment of patients with TB. Pyrazinamide (PZA) is an important first-line anti-TB drug used in combination with rifampicin, isoniazid and ethambutol forms the cornerstone of modern TB therapy. MDR-TB has recently become a serious public health problem..${ }^{3,4}$ It is recommended by the World Health Organization (WHO) for treatment of every TB patient. It is also used to treat MDR-TB patients. ${ }^{2}$

The PZA is an analogue of nicotinamide, which is a vitamin B3 (nicotinic acid; also called niacin) precursor. The discovery of PZA in the late 1940s as a powerful drug against TB was based on the serendipitous observation that nicotinamide, curiously, had activity against tubercle bacilli in animal models. Subsequent synthesis of analogues of nicotinamide led to the identification of PZA as the most active derivative against Mycobacterium tuberculosis (MTB) strains. ${ }^{3,5}$ Although PZA was synthesized before 1940s its anti-TB activity was not recognized until the early 1950 's. ${ }^{4}$ Despite many studies since its discovery 50years ago, the mechanism of action of PZA is the least understood of all TB drugs, because of its unusual properties. ${ }^{6}$

PZA plays a unique role in shortening the therapy from a period of $9-12$ months to 6 months, because it kills a population of semi-dormant tubercle bacilli, residing in an acidic environment (occurring during active inflammation), which are not killed by other TB drugs. Unlike other TB drugs, PZA, despites its remarkable activity in vivo, has no activity against tubercle bacilli in vitro in normal culture medium except under acidic-pH conditions (e.g. $\mathrm{pH} 5.5){ }^{3}$ Hence, PZA DST is technically difficult because it is active only at acid $\mathrm{pH},{ }^{7}$ however, the reasons why PZA requires an acidic environment to show activity is unknown. ${ }^{3}$ Unfortunately, the low $\mathrm{pH}$ (5.5) required itself prevents up to $50 \%$ of strains from growing in $7 \mathrm{H} 10$ Middle brook agar medium. Even with recent improvements in the use of this medium, about $10 \%$ of clinical isolates do not grow at $\mathrm{pH} 5.5$.?

Pyrazinamidase (PZase) (186 amino acid) enzyme is encoded by pncA (561bp) gene produced by MTB strains. Loss of PZase activity causes PZA resistance in MTB. PZA has no apparent activity against tubercle bacilli under normal conditions. Although its mechanism of action is least understood. It has been shown that acidic $\mathrm{pH}$ enhances the accumulation of Pyrazinoic acid (POA). Action of PZA depends on the $\mathrm{pH}$ as it is active only in acidic medium. ${ }^{5}$ Acquired resistance to PZA occurs concomitantly with loss of PZase activity. Due to mutation in pncA, the PZase activity is lost and bacteria become incapable of converting prodrug PZA to its bactericidal form, POA. Loss of PZase activity correlates with the development of PZA 
resistance in MTB strains. Research done on several sequenced pncA gene of PZA-resistant and sensitive isolates showed that $70-97 \%$ of the resistant isolates have mutations in the pncA gene or the upstream region. Hence, pncA gene encoding the PZase is the major mechanism of resistance as PZA- resistant clinical isolates of MTB are usually defective in PZase activity. ${ }^{5}$

PZA itself has no discernable activity against tubercle bacilli. PZA susceptible strains of MTB deaminate PZA to POA, the metabolite with anti-TB activity. PZA is converted to POA by mediation of PZase in MTB and it inhibits fatty acid synthesis. Reduced PZase activity correlates well with resistance to PZA. ${ }^{1}$ Therefore, determination of the activity of a given strain of MTB to elaborate PZase has been suggested as a rapid method for PZA susceptibility testing and for screening PZA-susceptible strains. Unfortunately, this method fails to give information regarding the proportion of a test population that is PZA resistant, and it is also independent of PZA concentration. ${ }^{\text {? }}$

MTB is uniquely susceptible to PZA, whose MIC for this bacterium is about $16-50 \mu \mathrm{g} / \mathrm{ml}$. In contrast, other mycobacteria, and all nontuberculous mycobacteria (NTM), are completely insensitive to PZA. In MTB, the susceptibility to PZA correlates with the presence of a single enzyme with nicotinamidase and PZase activities. Strains of MTB that are resistant to PZA are often defective in PZase activity. The mutations that confer PZA resistance have been investigated on the PZase gene (pncA). Thus, finding mutations on the pncA gene makes the rapid detection of PZA-resistant MTB possible. ${ }^{1}$ The identification of mutations within the pncA gene has been proposed as a surrogate marker for PZA resistance in MTB. ${ }^{8}$ Unfortunately, susceptibility testing for PZA is still not sufficiently standardized to help guide therapy. Accordingly, there is a major need for more rapid and reliable tests. One approach is to detect mutations in the pncA gene. It has been reported that these mutations correlate well with a MIC of PZA of $>100 \mu \mathrm{g} / \mathrm{ml}$, with the frequency of pncA mutation among resistant strains (depending on the geographic area) ranging from 66.7 to $96.8 \%{ }^{9}$

A variety of techniques can be used for testing anti-TB drugs other than PZA, including the agar proportion method in its direct and indirect versions. The only PZA test approved by Food and Drug administration in the United States is the radiometric method using the special PZA liquid medium at $\mathrm{pH} 6.0$ in the BACTEC-460 system. ${ }^{10}$ This reference method, used to detect resistance of clinical isolates of MTB to PZA is difficult to perform due to requirement of an acid $\mathrm{pH}$ in the medium where the growth of MTB is often inhibited. For this reason many laboratories do not perform DST for PZA. ${ }^{2}$ This method is quite expensive and may not be affordable for many laboratories, especially in developing countries. ${ }^{10}$

Resistance to any of the primary drugs, including PZA, makes the disease more difficult and expensive to treat. The rapid detection of these resistant isolates is critical to the effective patient management. Hence, the rapid as well as reliable DST for PZA is vital as it is administered in the early intensive phase of TB. PZA is a part of Directly Observed Therapy, short course (DOTS) recommended by WHO, reduces the length of a course from previous 9 to 12 months to 6months; thus, aiding compliance and decreasing the risk of development of MDR-TB. The drug has capability of killing nonreplicating cell populations in an acidic $\mathrm{pH}$ environments that are not killed by other TB drugs. ${ }^{6,11}$ It is paradoxical and unconventional drug. ${ }^{12}$ Despite its remarkable sterilizing activity in vivo, ${ }^{13,14}$ it is not active against M. tuberculosis under 'normal' culture conditions near neutral $\mathrm{pH} .{ }^{15}$ It is only active against MTB at acid $\mathrm{pH}^{16}$ an environment that is produced during active inflammation, and its activity is closely related to the acidity of the medium., ${ }^{7}$ Even at acid $\mathrm{pH}$ 5.6, PZA kills MTB slowly and incompletely with no more than $76 \%$ of the bacterial population being killed by $1000 \mathrm{mg} / \mathrm{L} \mathrm{PZA},{ }^{18}$ which is $10-12$ times higher than the already high minimum inhibitory concentration (MIC) of $50-100 \mathrm{mg} / \mathrm{L} .{ }^{12}$ Unlike conventional antibiotics, which are more effective against the actively growing bacteria than non-growing bacteria, PZA is exactly the opposite, that is, it is less active against young growing tubercle bacilli but is more active against old nongrowing bacilli. ${ }^{17}$ However, reliable testing of the susceptibility to MTB to PZA in vitro is challenging. ${ }^{11}$ The Bactec 460 TB radiometric system [Becton Dickinson (BD), Sparks MD,USA] and BactecmgIT 960 are the only two methods are recommended by the Clinical and Laboratory Standards Institute (CLSI) ${ }^{19}$ and WHO for susceptibility testing to PZA, due to the difficulty in standardizing its susceptibility on solid media. ${ }^{11}$ Most laboratories have now replaced the $460 \mathrm{~TB}$ system with no radiometric BactecmgIT 960 system. Both methods utilize an acidified Middle brook broth and a critical concentration of $100 \mu \mathrm{g} / \mathrm{ml} .{ }^{19}$ DST in such liquid media is costly, especially in some regions that do not have enough economic capabilities. ${ }^{20}$ Several other DST methods have been developed, including the molecular drug susceptibility test (mDST) based on the detection of a pncA mutation, the PZase activity assay, and colorimetric methods based on a MIC or redox indicator. ${ }^{21-26}$

In this study we enrolled 343 clinical MTB isolates to evaluate the two methods for determination of the PZA DST i.e. mDST for pncA whole gene sequencing by Sanger method ${ }^{27,28}$ and PZase enzyme activity assay by Wayne's method. ${ }^{29}$

Having this background in mind, we planned a study with aims and objectives as:-

I. To perform PZA drug susceptibility testing (DST) by Enzymatic Pyrazinamidase Assay using Wayne's method.

II. To detect pncA gene mutations in MTB isolates by the PCR mediated Direct DNA sequencing technique using Sanger method.

III. Compare and analyse both the techniques to find out sensitivity and specificity in accordance with PPV and NPV.

IV. To know the most suitable method in the resource constrained laboratories for detection of PZA resistance in MTB clinical isolates.

Inclusion criteria: Phenotypically identified pulmonary MTB isolates.

Exclusion criteria: Non-tuberculous Mycobacterial (NTM) isolates.

\section{Material and methods}

The study was conducted over a period of two and half years from January 2014 to June 2016 in the department of Microbiology, Grant Government Medical College and Sir J. J. Group of Hospitals in Mumbai and mDST was done in Intermediate Reference Laboratory, Govt. Hospital for Chest Diseases, Gorimedu, Puducherry.

All the 343 isolates of MTB fulfilling the inclusion criteria and recovered during the study period were taken. 
These isolates were grown on conventional solid culture medium Lowenstein-Jensen medium (LJ) were selected based on their growth characteristics such as rough, tough and buff (cauliflower type of colonies), crumbly, waxy and cream colored colonies. From all, colony smear were prepared, air-dried, heat-fixed and then stained by Ziehl-Neelsen (Z-N) method. ${ }^{30}$

Phenotypic and Genotypic identification of MTB and MTB complex isolates were done as follows:

Phenotypic identification: ${ }^{30-32}$ was carried out using following criteria.

A. Rate of growth, pigment production, nitrate, niacin \& catalase tests.

B. Inability to grow on LJ containing P-nitrobenzoate $(500 \mu \mathrm{g} / \mathrm{ml})$

PZA DST by Enzymatic Pyrazinamidase Assay using Wayne's method. ${ }^{31}$

PZase is an enzyme that deaminates PZA to form POA, which produces pink-red band in the culture medium. ${ }^{31}$

Wayne's Method: 29,31

Principle: The deamination of PZA to POA and ammonia in 4days is a useful phenotypic characteristic by which weakly niacin positive strains of M. bovis (negative) can be distinguished from MTB complex (positive)

Quality control:
PZA Susceptible Control: M. tuberculosis type strain H37Rv (ATCC 27294).

PZA Resistant Control: M. bovis type strain (ATCC 19210). Procured from National Tuberculosis Reference Laboratory (Beijing, China). ${ }^{11}$

PZase substrate medium:-

a. 6.5g of Dubos broth base (Difco Laboratories, Detroit, MI) ${ }^{11}$ dissolved in $1 \mathrm{~L}$ distilled water.

b. Following ingredients were added to above

1. Pyrazinamide $0.1 \mathrm{~g}$

2. Sodium pyruvate $2.0 \mathrm{~g}$

3. Agar $15 \mathrm{~g}$

c. Heated to dissolve. Then dispensed in $50.0 \mathrm{ml}$ amounts in to screw-cap tubes.

d. Then autoclaved for 15 minutes at $121^{\circ} \mathrm{C}$.

e. Then agar allowed to harden with tubes in upright position.

Reagents ( $1 \%$ ferrous ammonium sulfate):-It was prepared immediately before use.

1. Ferrous ammonium sulfate - 0.1 gm was placed in sterile screwcapped test tubes.

2. $10 \mathrm{ml}$ of sterile deionized water added immediately before use \& crystals allowed to dissolve.

\section{Surface inoculation of 2 set of medium with heavy loopful growth, incubate at $37^{\circ} \mathrm{C}$ for 4 days}<smiles>[C]1[C-]=C=C1</smiles>

Add $1 \mathrm{ml}$ freshly prepared $1 \%$ Ferrous Ammonium Sulphate reagent

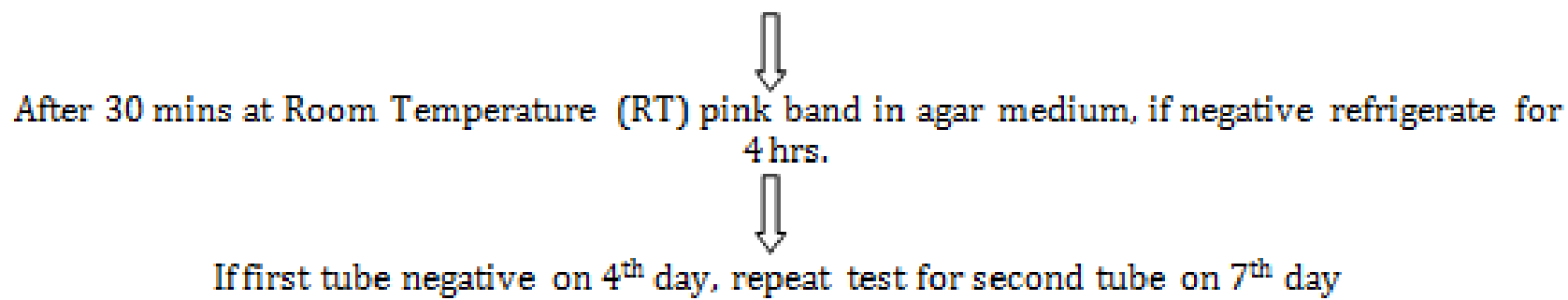

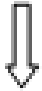

If $4^{\text {th }}$ day tube positive, $2^{\text {nd }}$ tube discarded without further confirmation

Interpretation $(\mathrm{N}=343)$ :

After 4hours, tubes were examined for pink band in reagent layer on the surface of agar (positive reaction), using incident room light against a white background. The procedure was repeated with remaining tubes after 7days of incubation. All tests were performed under a biologic safety hood.

Positive $(200 / 343$ i.e. $58.33 \%)=$ Pink band in reagent layer on agar surface i.e., PZA Sensitive/wild.

Negative $(143 / 343$ i.e. $41.69 \%)=$ No pink band (defective PZase activity) i.e. PZA Resistant/mutant.

Detection of pncA gene mutations by PCR mediated Direct DNA sequencing technique using Sanger method. This was carried out to detect pncA gene mutations in all 343 MTB clinical isolates as shown in following Figure. ${ }^{9,28,33}$ 


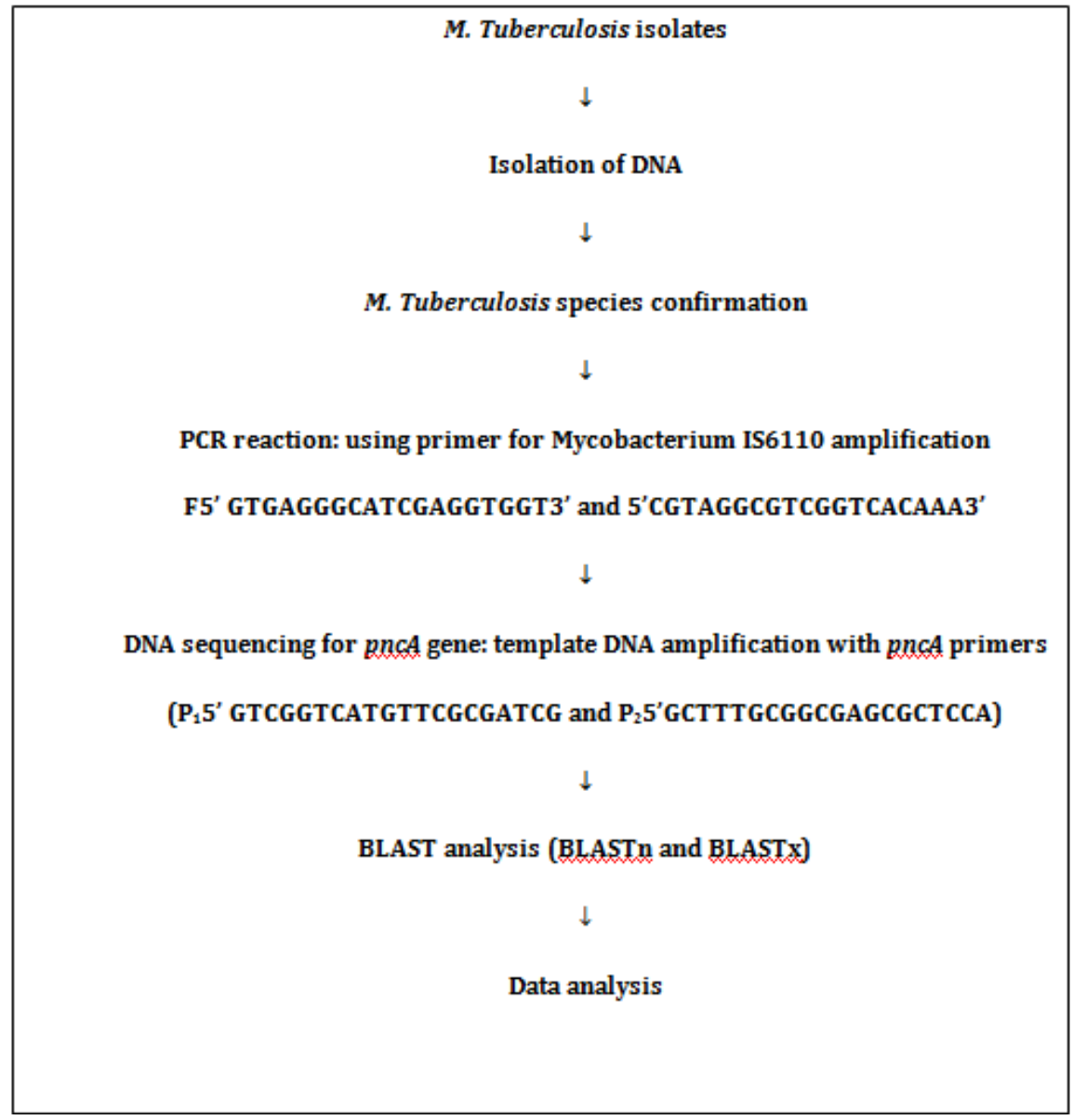

\section{Observation and results}

Wayne's and resistant by Sanger pncA sequencing with maximum in

51/110 (46.36\%) strains showed discordance as sensitivitePZA by

Table I Analysis of pncA mutations by Sanger sequencing vis a vis PZase activity by Wayne's assay $(N=5 I)$

\begin{tabular}{|c|c|c|c|c|c|}
\hline $\begin{array}{l}\text { Sr. } \\
\text { no. }\end{array}$ & $\begin{array}{l}\text { Strain } \\
\text { No. }\end{array}$ & $\begin{array}{l}\text { PPZase activity } \\
\text { assay }\end{array}$ & $\begin{array}{l}\text { mDST method based on detection of } \\
\text { pncA mutations }\end{array}$ & Frequency & \% Frequency \\
\hline I & 2016 & S & QI4IP & $\mathrm{FI}$ & 3.1 \\
\hline 2 & 1734 & S & GI32A & $\mathrm{FI}$ & 3.1 \\
\hline 3 & 1796 & S & V44D & $\mathrm{FI}$ & 3.1 \\
\hline 4 & 2385 & S & $\mathrm{s} 84 \mathrm{C}$ & $\mathrm{FI}$ & 3.1 \\
\hline 5 & 2423 & S & D86B & $\mathrm{FI}$ & 3.1 \\
\hline 6 & 2182 & S & R2G,EI8IR,LI82V & $\mathrm{FI}, \mathrm{FI}, \mathrm{FI}$ & 3.I,3.1,3.I \\
\hline 7 & 3478 & S & SI9IA, SI92A & $\mathrm{FI}, \mathrm{FI}$ & $3.1,3.1$ \\
\hline 8 & 2715 & S & $D I 2 A$ & $\mathrm{FI}$ & 3.1 \\
\hline 9 & 3560 & S & $15 \mathrm{~S}$ & $\mathrm{FI}$ & 3.1 \\
\hline 10 & 3083 & S & VI44A & $\mathrm{FI}$ & 3.1 \\
\hline 11 & 3274 & $S$ & $\begin{array}{l}\text { NIIK,DI2N,EI5K,A20W,G23R, } \\
\text { G24A,S35W,L39W,A40G,H46R, } \\
\text { H47R,V49T,V48S,T5IP,K52C, } \\
\text { D53Y,I56F,D57G,H6IG,F62V, } \\
\text { G64R,T65A,P66F,Y68L,S70T, } \\
\text { S7IT,H75Y,V77S,G79F,H86S,D90N }\end{array}$ & $\begin{array}{l}\mathrm{FI}, \mathrm{FI}, \mathrm{FI}, \mathrm{FI}, \mathrm{FI}, \mathrm{FI}, \mathrm{FI}, \mathrm{FI}, \mathrm{FI}, \mathrm{FI}, \mathrm{FI}, \\
\mathrm{FI}, \mathrm{FI}, \mathrm{FI}, \mathrm{FI}, \mathrm{FI}, \mathrm{FI}, \mathrm{FI}, \mathrm{FI}, \mathrm{FI}, \\
\mathrm{FI}, \mathrm{FI}, \mathrm{FI}, \mathrm{FI}, \mathrm{FI}, \mathrm{FI}, \mathrm{FI}, \mathrm{FI}, \mathrm{FI}, \mathrm{FI}, \mathrm{FI}\end{array}$ & 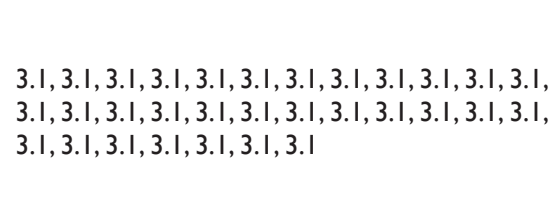 \\
\hline
\end{tabular}


Table Continued...

\begin{tabular}{|c|c|c|c|c|c|}
\hline $\begin{array}{l}\text { Sr. } \\
\text { no. }\end{array}$ & $\begin{array}{l}\text { Strair } \\
\text { No. }\end{array}$ & $\begin{array}{l}\text { nPZase activity } \\
\text { assay }\end{array}$ & $\begin{array}{l}\text { mDST method based on detection of } \\
\text { pncA mutations }\end{array}$ & Frequency & $\%$ Frequency \\
\hline 12 & 3410 & $\mathrm{~S}$ & H75Y,GII2R & $\mathrm{FI}, \mathrm{FI}$ & $3.1,3.1$ \\
\hline 13 & 2905 & $\mathrm{~S}$ & D53G & $\mathrm{FI}$ & 3.1 \\
\hline 14 & 4016 & $S$ & D53G,LI6IM & $\mathrm{FI}, \mathrm{FI}$ & $3.1,3.1$ \\
\hline 15 & 3658 & $\mathrm{~S}$ & VI44A & $\mathrm{FI}$ & 3.1 \\
\hline 16 & 2831 & $S$ & VI $44 A$ & $\mathrm{FI}$ & 3.1 \\
\hline 17 & 2420 & $S$ & SI92V & $\mathrm{FI}$ & 3.1 \\
\hline 18 & 3851 & $\mathrm{~S}$ & R2G,EI87R,LI88V & $\mathrm{FI}, \mathrm{FI}, \mathrm{FI}$ & 3.1 \\
\hline 19 & 3739 & $S$ & LI6IP & $\mathrm{FI}$ & 3.1 \\
\hline 20 & 3855 & $S$ & VI $44 A$ & $\mathrm{FI}$ & 3.1 \\
\hline 21 & 2776 & $\mathrm{~S}$ & AI06S,SI08Q,EIIIQ,GII2R,VII3I & $\mathrm{FI}, \mathrm{FI}, \mathrm{FI}, \mathrm{FI}, \mathrm{FI}$ & 3.I,3.I,3.I,3.I,3.I \\
\hline 22 & 3887 & $\mathrm{~S}$ & $\begin{array}{l}\text { A96G,V97G,F98V,Y99L,K I00Q,A I 02W, } \\
\text { Y I 03P,G I 05P,A I 06E,YI 07R,SI08T, } \\
\text { RI I OA,EI I IS,G I I 2R,VI I 3S,DI I 4R, } \\
\text { GI I 7R }\end{array}$ & $\begin{array}{l}\mathrm{FI}, \mathrm{FI}, \mathrm{FI}, \mathrm{FI}, \mathrm{FI}, \mathrm{FI}, \mathrm{FI}, \mathrm{FI}, \mathrm{FI}, \mathrm{FI} \\
\mathrm{FI}, \mathrm{FI}, \mathrm{FI}, \mathrm{FI}, \mathrm{FI}, \mathrm{FI}, \mathrm{FI}\end{array}$ & $\begin{array}{l}\text { 3.I, 3.I, 3.I, 3.I, 3.I, 3.I, } \\
\text { 3.I, 3.I, 3.I, 3.I, 3.I, 3.I, 3.I, 3.I, 3.I, 3.I, 3.I }\end{array}$ \\
\hline 23 & 2531 & $\mathrm{~S}$ & $\begin{array}{l}\text { A96G,F98S,Y99T,KI 00R,AI 02P,TI04R, } \\
\text { AI06D,DII4S,EII5R,NI I6D,LI22I, } \\
\text { NI23G }\end{array}$ & $\begin{array}{l}\mathrm{FI}, \mathrm{FI}, \mathrm{FI}, \mathrm{FI}, \mathrm{FI}, \mathrm{FI}, \mathrm{FI}, \mathrm{FI}, \mathrm{FI}, \mathrm{FI}, \\
\mathrm{FI}, \mathrm{FI}\end{array}$ & 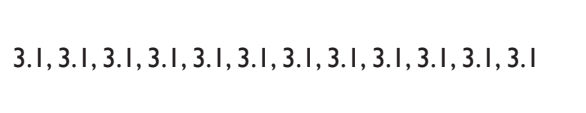 \\
\hline 24 & 3705 & $\mathrm{~S}$ & 194S,E95R,A96R,KI00Q & $\mathrm{FI}, \mathrm{FI}, \mathrm{FI}, \mathrm{FI}$ & 3.I, 3.I, 3.I, 3.1 \\
\hline 25 & 3856 & $\mathrm{~S}$ & 194S,E95R,A96R,KI00Q & $\mathrm{FI}, \mathrm{FI}, \mathrm{FI}, \mathrm{FI}$ & 3.I, 3.I, 3.I, 3.I \\
\hline 26 & 2672 & $\mathrm{~S}$ & $\begin{array}{l}\text { DI50T,AI5IR,VI52Y,NI54M,AI57G, } \\
\text { TI58H,RI59Q,SI70G,AI7IR,DI72Y, } \\
\text { TI73H,TI74R,AI76R,LI 78G,EI79R, } \\
\text { MI8IL,RI82A }\end{array}$ & $\begin{array}{l}\mathrm{FI}, \mathrm{FI}, \mathrm{FI}, \mathrm{FI}, \mathrm{FI}, \mathrm{FI}, \mathrm{FI}, \mathrm{FI}, \mathrm{FI}, \mathrm{FI}, \\
\mathrm{FI}, \mathrm{FI}, \mathrm{FI}, \mathrm{FI}, \mathrm{FI}, \mathrm{FI}, \mathrm{FI}\end{array}$ & $\begin{array}{l}\text { 3.I, 3.I, 3.I, 3.I, 3.I, 3.I, 3.I, 3.I, 3.I, 3.I, 3.I, 3.I, } \\
\text { 3.I, 3.I, 3.I, 3.I, 3.I }\end{array}$ \\
\hline 27 & 2837 & $\mathrm{~S}$ & $\mathrm{H} 75 \mathrm{Y}$ & $\mathrm{FI}$ & 3.1 \\
\hline 28 & 3323 & $\mathrm{~S}$ & W72L,P74Q,H75Q,A I06E,YI07N, LI2IM & $\mathrm{FI}, \mathrm{FI}, \mathrm{FI}, \mathrm{FI}, \mathrm{FI}, \mathrm{FI}$ & $3.1,3.1,3.1,3.1,3.1,3.1$ \\
\hline 29 & 2862 & $\mathrm{~S}$ & AI76V,EI87R,LI88V & $\mathrm{FI}, \mathrm{FI}, \mathrm{FI}$ & 3.I, 3.I, 3.I \\
\hline 30 & 3279 & $\mathrm{~S}$ & $\begin{array}{l}\text { MII,S36R,L39W,A40P,E4IK,A42R, } \\
\text { A43G,A50Q,T5IP,D53I,F54P,S57R, } \\
\text { T65H,P66T,D67G,Y68L,S69F,S70L, } \\
\text { S7IV,W72G }\end{array}$ & $\begin{array}{l}\mathrm{FI}, \mathrm{FI}, \mathrm{FI}, \mathrm{FI}, \mathrm{FI}, \mathrm{FI}, \mathrm{FI}, \mathrm{FI}, \mathrm{FI}, \mathrm{FI}, \\
\mathrm{FI}, \mathrm{FI}, \mathrm{FI}, \mathrm{FI}, \mathrm{FI}, \mathrm{FI}, \mathrm{FI}, \mathrm{FI}, \mathrm{FI}, \mathrm{FI}\end{array}$ & $\begin{array}{l}\text { 3.I, 3.I, 3.I, 3.I, 3.I, 3.I, 3.I, 3.I, 3.I, 3.I, 3.I, 3.I, } \\
\text { 3.I, 3.I, 3.I, 3.I, 3.I, 3.I, 3.I, 3.I }\end{array}$ \\
\hline 31 & 2678 & $\mathrm{~S}$ & GI7D,G24S,MII, & $\mathrm{FI}, \mathrm{FI}, \mathrm{FI}$ & 3.I, 3.I, 3.I \\
\hline 32 & 3264 & $\mathrm{~S}$ & TI47A & $\mathrm{FI}$ & 3.1 \\
\hline 33 & 3333 & $\mathrm{~S}$ & SI70L & $\mathrm{FI}$ & 3.1 \\
\hline 34 & 3931 & $\mathrm{~S}$ & VI44A & $\mathrm{FI}$ & 3.1 \\
\hline 35 & 3268 & $\mathrm{~S}$ & VI44A & $\mathrm{FI}$ & 3.1 \\
\hline 36 & 3035 & $\mathrm{~S}$ & P66L & $\mathrm{FI}$ & 3.1 \\
\hline 37 & 3009 & $\mathrm{~S}$ & Y68H,S7IL & $\mathrm{FI}, \mathrm{FI}$ & 3.I, 3.I \\
\hline 38 & 2860 & $\mathrm{~S}$ & L27P,D53B,TI74S,A I 77T,LI88F & $\mathrm{FI}, \mathrm{FI}, \mathrm{FI}, \mathrm{FI}, \mathrm{FI}$ & 3.I, 3.I, 3.I, 3.I, 3.1 \\
\hline 39 & 483 & $\mathrm{~S}$ & $\mathrm{D}|4| \mathrm{G}$ & $\mathrm{FI}$ & 3.1 \\
\hline 40 & 1104 & $S$ & $\begin{array}{l}\text { D60H,H6IQ,F62G,S63A,T65G,T80C, } \\
\text { P8IV,A83R,F84Y,P86R,Q I46R,TI47G, } \\
\text { AI48G,EI49G,VI52H,NI54Q,TI83R, } \\
\text { A I84V,SI85G,VI86L,EI87Q,VI89L, } \\
\text { LI90M, SI9IA, SI92P }\end{array}$ & $\begin{array}{l}\mathrm{FI}, \mathrm{FI}, \mathrm{FI}, \mathrm{FI}, \mathrm{FI}, \mathrm{FI}, \mathrm{FI}, \mathrm{FI}, \mathrm{FI}, \mathrm{FI}, \\
\mathrm{FI}, \mathrm{FI}, \mathrm{FI}, \mathrm{FI}, \mathrm{FI}, \mathrm{FI}, \mathrm{FI}, \mathrm{FI}, \mathrm{FI}, \mathrm{FI}, \\
\mathrm{FI}, \mathrm{FI}, \mathrm{FI}, \mathrm{FI}, \mathrm{FI}\end{array}$ & $\begin{array}{l}\text { 3.I, 3.I, 3.I, 3.I, 3.I, 3.I, 3.I, 3.I, 3.I, 3.I, 3.I, 3.I, } \\
\text { 3.I, 3.I, 3.I, 3.I, 3.I, 3.I, 3.I, 3.I, 3.I, 3.I, 3.I, 3.I, 3.I }\end{array}$ \\
\hline
\end{tabular}


Table Continued....

\begin{tabular}{|c|c|c|c|c|c|}
\hline $\begin{array}{l}\text { Sr. } \\
\text { no. }\end{array}$ & $\begin{array}{l}\text { Strain } \\
\text { No. }\end{array}$ & $\begin{array}{l}\text { nPZase activity } \\
\text { assay }\end{array}$ & $\begin{array}{l}\text { mDST method based on detection of } \\
\text { pncA mutations }\end{array}$ & Frequency & $\%$ Frequency \\
\hline 41 & 788 & $\mathrm{~S}$ & A24P,I25S,SI9IA,SI92A & $\mathrm{FI}, \mathrm{FI}, \mathrm{FI}, \mathrm{FI}$ & 3.I, 3.I, 3.I, 3.I \\
\hline 42 & 198 & $\mathrm{~S}$ & DI4IG & $\mathrm{FI}$ & 3.1 \\
\hline 43 & 780 & $\mathrm{~S}$ & D90H,RI54F,A I76D,A I76G,LI88V & $\mathrm{FI}, \mathrm{FI}, \mathrm{FI}, \mathrm{FI}, \mathrm{FI}$ & 3.I, 3.I, 3.I, 3.I, 3.I \\
\hline 44 & 759 & $\mathrm{~S}$ & $\begin{array}{l}\text { TI04H,GI05R,A I05R,AI06T,MIV, } \\
\text { Y99L,KI00Q,AI02C,YI03L }\end{array}$ & $\mathrm{FI}, \mathrm{FI}, \mathrm{FI}, \mathrm{FI}, \mathrm{FI}, \mathrm{FI}, \mathrm{FI}, \mathrm{FI}, \mathrm{FI}$ & 3.I, 3.I, 3.I, 3.I, 3.I, 3.I, 3.I, 3.I, 3.I \\
\hline 45 & $|72|$ & $S$ & MI8IR & $\mathrm{FI}$ & 3.1 \\
\hline 46 & 925 & $\mathrm{~S}$ & A83V,SI85Q & $\mathrm{FI}, \mathrm{FI}$ & $3.1,3.1$ \\
\hline 47 & 699 & $\mathrm{~S}$ & VI44A & $\mathrm{FI}$ & 3.1 \\
\hline 48 & 530 & $S$ & GI37A,SI70W & $\mathrm{FI}, \mathrm{FI}$ & 3.I, 3.I \\
\hline 49 & 485 & $\mathrm{~S}$ & $\begin{array}{l}\text { GI6V,T22P,CI4A,G23V,FI3S,L27P, } \\
\text { GI7A,SI8R,A20R }\end{array}$ & FI,FI, FI, FI, FI, FI, FI, FI, FI & 3.I, 3.I, 3.I, 3.I, 3.I, 3.I, 3.I, 3.I, 3.I \\
\hline 50 & 1722 & $\mathrm{~S}$ & SI9IK & $\mathrm{FI}$ & 3.1 \\
\hline 51 & 296 & $\mathrm{~S}$ & VI44G & $\mathrm{FI}$ & 3.1 \\
\hline
\end{tabular}

\section{Discussion}

Patient morbidity/mortality along with treatment-associated costs has been increased due to splurge in drug resistant TB. Hence rapid and accurate DST methods are required in the selection of appropriate TB drugs. However, "understanding drug resistance patterns within a given community is also of enormous epidemiological significance, since it provides indicators of the existence and prevalence of primary and acquired drug resistance which can be useful in the evaluation of the efficacy of anti-TB treatment. ${ }^{11}$ Several WHO-recommended DST methods are available for assessing drug resistance in the commonly used anti-TB drugs such as rifampin, isoniazid, ethambutol and streptomycin. These include solid LJ, liquid Middlebrook 7H10/ Middlebrook 7H11 and Bactec 460/Bactec 960 methods. However, for PZA which is one of the most important early oral anti-TB agents, WHO recommended only Bactec 460 and Bactec 960 methods. $^{20}$ The main reason for this is that many of The other methods of PZA drug susceptibility testing have disadvantages, such as slow and poor growth as well as difficult standardization that makes them unsuitable for use in clinical mycobacteriology laboratories. These tests are colorimetric methods based on redox indicators and MIC, PZase activity assays and $\mathrm{mDST}$ based on the detection of pncA mutations. Studies conducted on the performances of these methods have been reported. ${ }^{21-26}$ However, studies on the use of these methods or comparing several methods synchronously using large quantities of clinically isolated MTB strains for PZA drug susceptibility been reported. ${ }^{11}$

For PZA susceptibility testing Radiometric BactecmgIT 960 method that utilize an acidified Middlebrook broth with a critical concentration of $100 \mu \mathrm{g} / \mathrm{ml}^{19}$ is commonly used method in developed countries. However, PZase enzyme activity assay using Wayne's method can be used as an alternative to BACTEC especially in areas where BACTEC is not available. ${ }^{29}$ PZA DST in such liquid media is costly, especially in those regions which are economically backward. ${ }^{20}$ Both Wayne's assay and Sanger mDST are based on the assumption that PZA-susceptible MTB strains produces PZase enzyme that hydrolyzes PZA drug into its active antibacterial moiety: POA. ${ }^{34}$ $p n c A$ is a gene that encodes PZase enzyme in MTB. Previous studies verified the fact that the mutation in pncA can inactivate PZase and confer resistance to PZA. ${ }^{35} \mathrm{Cui} \mathrm{Z}$ study ${ }^{11}$ indicated that the assumption regarding the mechanism of MTB resistance to PZA based on PZase did not explain all of the resistance mechanisms of MTB to PZA. Authors showed that 9.5\% (16/169) of the PZA-resistant strains were determined to be positive to PZase activity testing, indicating that, for these strains, PZA resistance was not caused by the inactivation of PZase but by some other unknown cause(s). ${ }^{11}$ Other studies also detected PZase activity in PZA-resistant strains. ${ }^{24,36,37}$ Cui Z study showed only $1 \%(3 / 260)$ of the PZA-susceptible strains negative according to the Wayne's assay, probably due to the weak activity of PZase or to the small quantity of MTB, which makes it difficult to observe the Wayne assay color change with the naked eye at 10days. ${ }^{11}$

Recently, Zhou et al., ${ }^{38}$ reported a new PCR-based in vitrosynthesized PZase method. This method might exclude false negatives from the PZase activity assay due to the small quantity of MTB. However, this method does not solve the false susceptibility of the PZase activity assay. False susceptibility is the major limitation of the Wayne's assay and should be considered seriously in clinical settings. ${ }^{11}$

The mDST by Sanger is an indirect method of assessing PZA susceptibility. PZase is inferred by the assessment of changes in the sequence of $p n c A$. If no amino acid substitution is detected in the coding sequence and no base substitution is detected in the promoter sequence, PZase is considered active; otherwise, PZase is considered inactive or absent. ${ }^{11}$ In Cui $\mathrm{Z}$ study, 9\% of the strains with the wildtype pncA gene (16/169) were found to be resistant to PZA, including 8 strains that tested positive for PZase activity and 8 strains that tested negative by the PZase activity assay. Hence we supports the results of Cui $\mathrm{z}$ et al that suggests that there might be other genes that regulate the expression of PZase and/or other unknown mechanisms for PZA resistance. ${ }^{11}$ As Cui $\mathrm{Z}$ reported amino acid substitutions in the pncA genes of PZA-susceptible strains. Most of these strains were positive for PZase activity. ${ }^{11}$ According to previous studies, mutations at certain amino acid sites, such as codons $8,96,134$, and 138, at active sites, and codons $13,49,51,57$, and 68 , coordinated to the $\mathrm{Fe}^{2+}$ ion, are closely associated with MTB resistance to PZA. ${ }^{39,40}$ Mutations at these sites can cause MTB to lose PZase activity and render the strain resistant to PZA. In the study by Cui Z, most amino acid substitutions in PZA-susceptible strains were far from the functional sites, and almost all of the amino acid substitutions occurred in PZA- 
susceptible strains. For this reason, Cui $\mathrm{Z}$ hypothesized that these amino acid substitutions did not lead to the loss of PZase activity. ${ }^{11}$ The in vitro-synthesized PZase assay can evaluate the correlation between the amino acid substitutions and PZase activity. If the amino acid substitutions far from the activity sites of PZase can be confirmed to be irrelevant to MTB resistance to PZA, then the specificity of the mDST can be improved. Additionally, Zhang and coworkers ${ }^{41}$ found that POA binds to RpsA, subsequently inhibiting trans-translation rather than canonical translation. They found that the deletion of amino acid 438 in the $\mathrm{C}$ terminus of RpsA can cause resistance to PZA in a clinical MTB isolate lacking pncA mutations.
Cui $\mathrm{Z}^{11}$ did not detect the deletion of amino acid 438 in the $\mathrm{C}$ terminus of RpsA and did not find any other mutation in the $\mathrm{C}$ terminus of RpsA. This suggests that the mutation rate of this site in the RpsA gene in clinical MTB isolates is too low to be a detection target for mDST. The sensitivities of the Wayne's assay and the Sanger mDST test should be improved to further clarify what the mechanism of PZA resistance is. ${ }^{11}$ We reported the sensitivity and specificity of Wayne's assay as $28.37 \%$ and $37.78 \%$, respectively, with PPV of $41.26 \%$ and NPV of $25.50 \%$ when tested against gold standard mDST Sanger method of pncA gene sequencing as shown in following Table 2.

Table 2 Comparison of Wayne's Assay vs. Sanger Sequencing

\begin{tabular}{lll}
\hline Isolates (N=343) & Sanger method (S) 233 (67.93\%) & Sanger method (R) I I (32.06\%) \\
\hline Wayne's method (S) & 149 & 51 \\
Wayne's method ( R) & 84 & 59 \\
Sensitivity & $28.37 \%$ & \\
Specificity & $37.78 \%$ & \\
PPV & $41.26 \%$ & \\
NPV & $25.50 \%$ & \\
\hline
\end{tabular}

The Sanger mDST satisfy the rapid test requirements and functions using smaller quantities of MTB than some other types of high-end laboratory equipment. In addition, the Wayne's assay requires a larger quantity of bacteria and does not take significantly less time, so there is no obvious advantage to using the Wayne's assay for the assessment of PZA susceptibility. ${ }^{11}$ There is no direct correlation between loss of PZase activity and the level of PZA resistance. ${ }^{37}$

Our observations suggest that, Wayne's method is rapid, technically easy to perform, cost effective and feasible. Though, Sanger method is an accurate, reliable, rapid gold standard; is expensive, needs specific infrastructure, equipment's and technical expertise. The comparative analysis of both techniques showed a sensitivity/specificity in accordance with worldwide literature.

\section{Conclusion}

Wayne's Method: Enzymatic Pyrazinamidase Assay can be used as a screening test in those laboratories those are not equipped for molecular testing but doing solid culture for detection of resistance to PZA in MTB isolates.

\section{Acknowledgements}

None.

\section{Conflict of interest}

The author declares no conflict of interest.

\section{References}

1. Suzuki Y, Suzuki A, Tamaru A, et al. Rapid detection of pyrazinamideresistant Mycobacterium tuberculosis by a PCR-based in vitro system. $J$ Clin Microbiol. 2002;40(2):501-507.

2. Martin A, Cubillos-Ruiz A, Von Groll A, et al. Nitrate reductase assay for the rapid detection of pyrazinamide resistance in Mycobacterium tuberculosis using nicotinamide. $J$ Antimicrob Chemother. 2008;61(1):123-127.

3. Zhang Y, Scorpio A, Nikaido H, et al. Role of acid $\mathrm{pH}$ and deficient efflux of pyrazinoic acid in unique susceptibility of Mycobacterium tuberculosis to pyrazinamide. J Bacteriol. 1999;181(7):2044-2049.
4. Salfinger M, Crowle AJ, Reller LB. Pyrazinamide and pyrazinoic acid activity against tubercle bacilli in cultured human macrophages and in the BACTEC system. J Infect Dis. 1990;162(1):201-207.

5. Singh $\mathrm{P}$, Mishra AK, Malonia SK, et al. The paradox of pyrazinamide: an update on the molecular mechanisms of pyrazinamide resistance in Mycobacteria. J Commun Dis. 2006;38(3):288-298.

6. Zhang Y, Wade MM, Scorpio A, et al. Mode of action of pyrazinamide: disruption of Mycobacterium tuberculosis membrane transport and energetics by pyrazinoic acid. J Antimicrob Chemother. 2003;52(5):790 795

7. Salfinger M, Heifets LB. Determination of pyrazinamide MICs for Mycobacterium tuberculosis at different $\mathrm{pHs}$ by the radiometric method. Antimicrob Agents Chemother. 1988;32(7):1002-1004.

8. Morlock GP, Crawford JT, Butler WR, et al. Phenotypic characterization of pncA mutants of Mycobacterium tuberculosis. Antimicrob Agents Chemother. 2000;44(9):2291-2295.

9. Huang TS, Lee SS, Tu HZ, et al. Correlation between pyrazinamide activity and pncA mutations in Mycobacterium tuberculosis isolates in Taiwan. Antimicrob Agents Chemother. 2003;47(11):3672-3673.

10. Heifets L, Sanchez T. New agar medium for testing susceptibility of Mycobacterium tuberculosis to pyrazinamide. J Clin Microbiol. 2000;38(4):1498-1501.

11. Cui Z, Wang J, Lu J, et al. Evaluation of Methods for Testing the Susceptibility of Clinical Mycobacterium tuberculosis Isolates to Pyrazinamide. J Clin Microbiol. 2013;51(5):1374-1380.

12. Zhang, Y, Mitchison DA. The curious characteristics of pyrazinamide: a review. Int J Tuberc Lung Dis. 2003;7(1):6-21.

13. McCune RM, Tompsett R, McDermott W. The fate of Mycobacterium tuberculosis in mouse tissues as determined by the microbial enumeration technique. II. The conversion of tuberculous infection to the latent state by administration of pyrazinamide and a companion drug. J Exp Med. 1956;104(5):763-802.

14. Grosset J. The sterilizing value of rifampicin and pyrazinamide in experimental short course chemotherapy. Bull Int Union Tuberc. 1978;53(1):5-12.

15. Tarshis MS, Weed WA. Lack of significant in vitro sensitivity of Mycobacterium tuberculosis to pyrazinamide on three different solid media. American Review of Tuberculosis. 1953;67:391-395 
16. McDermott $\mathrm{W}$, Tompsett $\mathrm{R}$. Activation of pyrazinamide and nicotinamide in acidic environment in vitro. Am Rev Tuberc. 1954;70(4):748-754.

17. Zhang Y, Permar S, Sun Z. Conditions that may affect the results of susceptibility testing of Mycobacterium tuberculosis to pyrazinamide. $J$ Med Microbiol. 2002;51(1):42-49.

18. Heifets LB, Lindholm-Levy PJ. Is pyrazinamide bactericidal against Mycobacterium tuberculosis? Am Rev Respir Dis. 1990141:250-252.

19. Chedore P, Bertucci L, Wolfe J, et al. Potential for Erroneous Results Indicating Resistance When Using the BACTEC MGIT 960 System for Testing Susceptibility of Mycobacterium tuberculosis to Pyrazinamide. $J$ Clin Microbiol. 2010;48(1):300-301.

20. World Health Organization. Guidelines for surveillance of drug resistance in tuberculosis. 4th ed. Switzerland: World Health Organization; 2009.

21. Denkin S, Volokhov D, Chizhikov V, et al. Microarray-based pncA genotyping of pyrazinamide-resistant strains of Mycobacterium tuberculosis. J Med Microbiol. 2005;54(Pt 12):1127-1131.

22. Campell PJ, Morlock GP, Sikes RD, et al. Molecular detection of mutations associated with first and second-line drug resistance compared with conventional drug susceptibility testing of Mycobacterium tuberculosis. Antimicrob Agents Chemother. 2011;55(5):2032-2041.

23. Miller MA, Thibert L, Desjardins F, et al. Testing of susceptibility of Mycobacterium tuberculosis to pyrazinamide: comparison of Bactec method with pyrazinamidase assay. J Clin Microbiol. 1995;33(9):24682470 .

24. Singh P, Wesley C, Jadaun GP, et al. Comparative evaluation of Lowenstein-Jensen proportion method, BacT/ALERT 3D system, and enzymatic pyrazinamidase assay for pyrazinamide susceptibility testing of Mycobacterium tuberculosis. J Clin Microbiol. 2007;45(1):76-80.

25. Leite CQ, Beretta AL, Anno IS, et al. Standardization of broth microdilution method for Mycobacterium tuberculosis. Mem Inst Oswaldo Cruz. 2000;95(1):127-129.

26. Syre H, Ovreas K, Grewal HM. Determination of the susceptibility of Mycobacterium tuberculosis to pyrazinamide in liquid and solid media assessed by a colorimetric nitrate reductase assay. J Antimicrob Chemother. 2010;65(4):704-712.

27. Prabhu SS, Muthuraj M, Muralidhar, et al. Rapid Detection of Pnca Mutations in Pyrazinamide-Resistant Mycobacterium tuberculosis Clinical isolates. European Journal of Applied Sciences. 2009;1(2):20 25.

28. Muthaiah M, Jagadeesan S, Ayalusamy N, et al. Molecular Epidemiological Study of Pyrazinamide-Resistance in Clinical Isolates of Mycobacterium tuberculosis from South India. Int J Mol Sci. 2010;11(7):2670-2680.
29. Krishnamurthy A, Almeida D, Rodrigues C, et al. Comparison of pyrazinamide drug susceptibility of $M$. tuberculosis by radiometric BACTEC and enzymatic pyrazinamidase assay. Indian J Med Microbiol. 2004;22(3):166-168.

30. Central TB Division. RNTCP Manual of Standard Operating Procedures (SOPs) for Culture of Mycobacterium Tuberculosis and Drug Susceptibility testing on Solid Medium. India: Intermediate Reference Laboratory for Tuberculosis; 2009.

31. Winn WC, Allen SD, Janda WM, et al. Mycobacteria. In: colour Atlas and Textbook of Diagnostic Microbiology. 6th ed. USA: Williams and Wilkins Publication, Lippincott; 2006. p. 1065-1117.

32. Colle JG, Duguid JP, Fraser AG, Marmion BP Mycobacterium: Tubercle bacilli. In: Mackie, McCartney Practical Medical Microbiology International Student. 14th ed. Singapore: Churchill Livingstone Publication; 1996. p. 329-341.

33. Wabale VR, Joshi AA, Muthaiah M, et al. pncA gene sequence analysis for pyrazinamide resistance in Mycobacterium tuberculosis from highincidence setting. Int J Pharm Bio Sci. 2016;7(4):648-654.

34. Heifets LB, Flory MA, Lindholm-Levy PJ. Does pyrazinoic acid as an active moiety of pyrazinamide have specific activity against Mycobacterium tuberculosis? Antimicrob. Agents Chemother. 1989;33(8):1252-1254.

35. Sun Z, Scorpio A, Zhang Y. The $p n c A$ gene from naturally pyrazinamideresistant Mycobacterium avium encodes pyrazinamidase and confers pyrazinamide susceptibility to resistant $M$. tuberculosis complex organisms. Microbiology. 1997;143:3367-3373.

36. Sharma B, Pal N, Malhotra B, et al. Comparison of MGIT 960 \& pyrazinamidase activity assay for pyrazinamide susceptibility testing of Mycobacterium tuberculosis. Indian J Med Res. 2010;132:72-76.

37. Butler WR, Kilburn JO. Susceptibility of Mycobacterium tuberculosis to pyrazinamide and its relationship to pyrazinamidase activity. Antimicrob Agents Chemother. 1983;24(4):600-601.

38. Zhou M, Geng X, Chen J, et al. Rapid colorimetric testing for pyrazinamide susceptibility of $M$. tuberculosis by a PCR-based in-vitro synthesized pyrazinamidase method. PLoS One. 2011;6(11):e27654

39. Quiliano M, Gutierrez AH, Gilman RH, et al. Structure-activity relationship in mutated pyrazinamidases from Mycobacterium tuberculosis. Bioinformation. 2011;6(9):335-339.

40. Petrella S, Gelus-Ziental N, Maudry A, et al. Crystal structure of the pyrazinamidase of Mycobacterium tuberculosis: insights into natural and acquired resistance to pyrazinamide. PLoS One. 2011;6(1):e15785.

41. Shi W, Zhang X, Jiang X, et al. Pyrazinamide inhibits trans-translation in Mycobacterium tuberculosis. Science. 2011;333(6049):1630-1632. 